NASA Technical Memorandum 106809

\title{
Completed Beltrami-Michell Formulation for Analyzing Mixed Boundary Value Problems in Elasticity
}

Surya N. Patnaik and Igor Kaljevic Ohio Aerospace Institute Brook Park, Ohio

Dale A. Hopkins

Lewis Research Center

Cleveland, Ohio

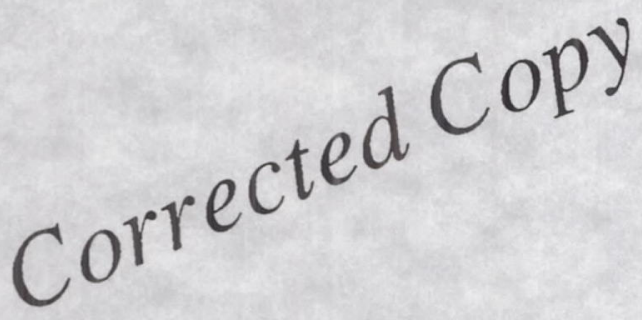

Sunil Saigal

Carnegie Mellon University

Pittsburgh, Pennsylvania

September 1995

National Aeronautics and Space Administration 


\title{
Completed Beltrami-Michell Formulation for Mixed \\ Boundary Value Problems in Elasticity
}

\author{
Surya N. Patnaik and Igor Kaljevic \\ Ohio Aerospace Institute \\ 22800 Cedar Point Road \\ Brook Park, OH 44142 \\ Dale A. Hopkins \\ National Aeronautics and Space Administration \\ Lewis Research Center \\ 21000 Brookpark Road, Mail Stop 49-8 \\ Cleveland, $\mathrm{OH} 44135$ \\ and \\ Sunil Saigal \\ Carnegie Mellon University \\ Department of Civil Engineering \\ Pittsburgh, PA 15213
}

\begin{abstract}
In elasticity, the method of forces, wherein stress parameters are considered as the primary unknowns, is known as the Beltrami-Michell Formulation (BMF). The existing BMF can only solve stress boundary value problems; it cannot handle the more prevalent displacement or mixed boundary value problems of elasticity. Therefore, this formulation, which has restricted application, could not become a true alternative to the Navier's displacement method, which can solve all three types of boundary value problems. The restrictions in the BMF have been alleviated by augmenting the classical formulation with a novel set of conditions identified as the boundary compatibility conditions. This new method, which completes the classical force formulation, has been termed the Completed Beltrami-Michell Formulation (CBMF). The CBMF can solve general elasticity problems with stress, displacement, and mixed boundary conditions in terms of stresses as the primary unknowns. The CBMF is derived from the stationary condition of the variational functional of the Integrated Force Method. In the CBMF, stresses for kinematically stable structures can be obtained without any reference to the displacements either in the field or on the boundary. This paper presents the CBMF and its derivation from the variational functional of the Integrated Force Method. Several
\end{abstract}


examples are presented to demonstrate the applicability of the completed formulation for analyzing mixed boundary value problems under thermomechanical loads. Selected example problems include a cylindrical shell, wherein membrane and bending responses are coupled, and a composite circular plate.

\section{Nomenclature}

$\boldsymbol{A}$

strain energy in the Integrated Force Method (IFM) functional

$a$

radius of shell

$B$

complementary strain energy in the IFM functional

$E$

modulus of elasticity

$[G]$

material matrix

h

plate or shell thickness

$\boldsymbol{K}$

plate or shell rigidity

$L$

length of shell

$M_{r}, M_{\varphi} \quad$ plate bending moments

$M_{x} \quad$ shell bending moment

$N_{\varphi} \quad$ shell tangential force

$n_{x}, n_{y} \quad$ directional cosines of the outward normal

$P_{x}, P_{y} \quad$ components of surface tractions

$q \quad$ intensity of the distributed load

$r, r_{a}, r_{b} \quad$ radial coordinates

$R(\sigma) \quad$ boundary compatibility condition in terms of stresses

$S_{1}(\sigma), S_{2}(\sigma) \quad$ traction conditions in terms of stresses

$t_{o} \quad$ temperature at the midsurface

$u, v, w \quad$ transverse displacement components

$\bar{u}, \bar{v} \quad$ prescribed boundary displacements

W potential of external loads in the IFM functional 


$\begin{array}{ll}x, y & \text { Cartesian coordinates } \\ x_{a}, x_{b} & \text { coordinates of shell contours } \\ \alpha_{t} & \text { coefficient of thermal expansion } \\ \beta & \text { cylindrical shell parameter } \\ \Gamma & \text { boundary of an elastic continuum } \\ \Delta t & \text { temperature difference between inner and outer surfaces } \\ \varepsilon_{x}, \varepsilon_{y}, \gamma_{x y} & \text { plane strain components } \\ v & \text { Poisson's ratio } \\ \Pi_{p}^{p s}, \Pi_{p}^{c}, \Pi_{s}^{c} & \text { IFM variational functional for plane stress, plates, and shells, respectively } \\ \sigma_{x}, \sigma_{y}, \tau_{x y} & \text { plane stress components } \\ \Phi & \text { Airy's stress function for plane stress } \\ \Psi & \text { shell stress function } \\ \Psi_{p}^{(q)}, \Psi_{p}^{(\Delta t)} & \text { particular integral for mechanical and thermal loads, respectively } \\ \Omega & \text { shell domain }\end{array}$

Introduction

The method of forces, also known as the Beltrami-Michell Formulation (BMF), and its variant, the Airy's stress function formulation, were the preferred tools of analysis in elasticity during the $1940^{\prime} \mathrm{s}$ and $1950^{\prime} \mathrm{s} .^{1,2}$ In fact, solutions for many classical elasticity problems have been obtained via the method of forces. ${ }^{1-3}$ The method of forces, however, could not compete with the Navier's displacement formulation, especially in analyzing plates and shells with displacement and mixed boundary conditions. Thus, the application of the method of forces diminished, and the displacement formulation gained popularity. The demise of the method of forces was not due to any intrinsic generic deficiency of the method but to the incompleteness of the formulation. Because a set of boundary equations was missing, the application of the classical BMF was restricted to solving only problems with stress boundary conditions. In other words, the Beltrami-Michell's force formulation can be used to solve stress boundary value problems, but it cannot solve the more prevalent displacement and mixed boundary value problems. The 
missing set of equations, which completes the BMF, has been identified as the boundary compatibility conditions. At this time, these boundary compatibility conditions have been derived only from a variational formulation. Direct derivation of boundary compatibility conditions is not known, and this may be the primary reason why these equations were not formulated earlier. Augmentation of the classical BMF with these boundary compatibility conditions resulted in a novel force method-the Completed Beltrami-Michell Formulation (CBMF). The CBMF bestows equal emphasis on stress equilibrium and strain compatibility conditions. It is as universal as the Navier's displacement formulation, solving all three classes of elasticity problems: stress, displacement, and mixed boundary value problems. Thus, the CBMF overcomes the deficiency of the classical BMF. The CBMF can provide solution to stresses without any reference to the displacements, either in the field or on the boundary, for kinematically stable structures.

The primary purpose of the structural analysis is to determine the internal stress state in an elastic continuum. In the CBMF, stresses are obtained directly as a solution to a set of equations of this formulation. Displacements, if required, can be calculated from stresses using integration. In the Navier's displacement method, displacements (whether required or not) must be generated first; then stresses are determined indirectly through differentiation. As a result, in the displacement method, stresses can become inaccurate, especially when approximate techniques are used. In the CBMF, problems with thermal and initial strains are handled directly by the compatibility formulation, whereas in the Navier's displacement method, they have to be treated indirectly using the concept of equivalent loads. The development of the CBMF is further justified because all the solutions that have been obtained with the classical BMF have to be verified; that is, it must be determined whether the boundary compatibility conditions have been satisfied or not. The noncompliance of boundary compatibility conditions for a classical elasticity solution is indicated in Ref. 4.

The novel boundary compatibility conditions, the key ingredient in the CBMF, were accidentally derived and then identified during the formulation of the variational functional ${ }^{5}$ of the Integrated Force Method (IFM) for the finite element discrete analysis. The IFM for the finite element analysis, which can be considered the discretized version of the CBMF, actually was formulated before the CBMF. Henceforth, in this paper, the force method for analyzing boundary value problems in elasticity and for analyzing plates and shells is called the Completed Beltrami-Michell Formulation (CBMF). The method of forces for the finite element numerical analysis is still referred to as the Integrated Force Method (IFM).

The boundary compatibility conditions were reported earlier for two-dimensional elasticity problems, ${ }^{5}$ and stress analyses using boundary compatibility conditions were published for rectangular ${ }^{4}$ and circular plates ${ }^{6}$ in flexure for mechanical loads. 
This paper includes the formal presentation of the CBMF and its application to analyzing circular plates and circular cylindrical shells subjected to both mechanical and thermal loads. Specialized equations for plates and shells are derived from the stationary condition of the IFM variational functional, and several mixed boundary value problems are solved to demonstrate the capability of the formulation. The first problemis a circular plate made of two different materials and subjected to thermomechanical loads. The solution of the plate example demonstrates the application of the CBMF to problems with displacement and interface (or jump) boundary conditions. Cylindrical shells are analyzed next. The shell examples demonstrate the use of the CBMF when membrane and bending responses are coupled. In addition, this paper serves as an initial, yet an unified and systematic, attempt to bring back the method of forces for analyzing general elastic continua. It is anticipated that the development of the CBMF for various shell structures, wherein membrane and bending responses are coupled, may become a significant avenue for research.

\section{Completed Beltrami-Michell Formulation of Elasticity}

The basic concepts of the method of forces (the CBMF being its specialization for analyzing elastic continua) can be initiated from the stress-strain law, which is universal to all analysis formulations. The stress-strain law that links stresses $\{\sigma\}$ to strains $\{\varepsilon\}$ through a known material matrix $[G]$ can be written as

$$
\{\sigma\}=[G]\{\varepsilon\}
$$

The stresses in Eq. (1) must satisfy the state of equilibrium, and the strains must satisfy compatibility conditions. In other words, the stresses in the method of forces can be determined from the stress strain law given by Eq. (1) and (I) the stress equilibrium equations and (II) the strain compatibility conditions. Displacements are not essential for the determination of stresses.

A finite elastic continuum consists of a field and a boundary. Stresses and strains must satisfy equilibrium equations and compatibility conditions both in the field and on the boundary, respectively, as

(Ia) Stress equilibrium equations in the field

(Ib) Stress equilibrium equations on the boundary (or traction conditions)

(IIa) Strain compatibility conditions in the field

(IIb) Strain compatibility conditions on the boundary 
In the method of forces, all equation sets ( $\mathrm{Ia}, \mathrm{Ib}, \mathrm{Ila}$, and $\mathrm{IIb})$, including the compatibility conditions, are expressed in terms of stresses. The equation set of the classical BMF contained conditions ( $\mathrm{Ia}$, Ib, and IIa), but it missed the boundary compatibility conditions (IIb). The CBMF utilizes all four conditions ( $\mathrm{Ia}, \mathrm{Ib}, \mathrm{II}$, and IIb).

\section{Governing Equations for the Completed Beltrami-Michell Formulation}

Consider the CBMF equations in the following plane stress problem. For simplicity and clarity, homogeneous kinematic boundary conditions are considered, and initial deformations along with body forces are neglected. The derivation of the equations from the IFM variational functional for nonhomogeneous boundary conditions with body forces is given in Ref. 5 and is not repeated here. However, a brief presentation of the IFM variational functional is provided in the appendix for quick reference. The equations, as obtained from the IFM functional, can be separated into five groups (Ia, Ib, IIa, IIb, and III) as follows:

\section{Group Ia: Equilibrium Equations in the Field}

$$
\begin{aligned}
& \frac{\partial \sigma_{x}}{\partial x}+\frac{\partial \tau_{x y}}{\partial y}=0 \\
& \frac{\partial \tau_{x y}}{\partial x}+\frac{\partial \sigma_{y}}{\partial y}=0
\end{aligned}
$$

Group Ib: Boundary Equilibrium Equations (or Traction Conditions)

$$
\begin{aligned}
& S_{1}(\sigma)=\sigma_{x} n_{x}+\tau_{x y} n_{y}-P_{x}=0 \\
& S_{2}(\sigma)=\tau_{x y} n_{x}+\sigma_{y} n_{y}-P_{y}=0
\end{aligned}
$$

where $\sigma_{x}, \sigma_{y}$, and $\tau_{x y}$ are three components of the stress tensor; $n_{x}$ and $n_{y}$ are the direction cosines of the outward normal vector; and $P_{x}$ and $P_{y}$ are prescribed boundary tractions. In the field, the equilibrium equations are functionally indeterminate ${ }^{7}$ because three unknown stresses are expressed in terms of two (Group Ia) equations.

\section{Group Ila: Field Compatibility Condition}

The functional indeterminacy in the domain is alleviated through the field compatibility condition of St. Venant, which can be written in terms of the strain components as 


$$
\frac{\partial^{2} \varepsilon_{y}}{\partial x^{2}}+\frac{\partial^{2} \varepsilon_{x}}{\partial y^{2}}-\frac{\partial^{2} \gamma_{x y}}{\partial x \partial y}=0
$$

and in terms of the stresses as

$$
\nabla^{2}\left(\sigma_{x}+\sigma_{y}\right)=0
$$

Equations (2), (3), and (5), in essence, represent the stress or the classical BMF in elasticity that was developed in $1900 .^{1}$ This formulation, which is incomplete, can only solve stress boundary value problems.

\section{Group IIb: Boundary Compatibility Condition}

Three stresses on the boundary are expressed in terms of two traction equations, Eqs. (3a) and (3b), thus, there is one degree of functional indeterminacy. The field compatibility condition given in Eq. (5) alleviated functional indeterminacy in the field. However, because St. Venant did not formulate the compatibility on the boundary, the stresses there remained indeterminate. The functional indeterminacy on the boundary, which made the Beltrami-Michell stress formulation incomplete, was alleviated by Patnaik ${ }^{5}$ with the formulation of the boundary compatibility condition. This boundary condition, when expressed in terms of stresses for isotropic material, has the following form

$$
\Phi(\sigma)=\frac{\partial}{\partial x}\left(\sigma_{y}-v \sigma_{x}\right) n_{x}+\frac{\partial}{\partial y}\left(\sigma_{x}-v \sigma_{y}\right) n_{y}-(1+v)\left(\frac{\partial \tau_{x y}}{\partial x} n_{y}+\frac{\partial \tau_{x y}}{\partial y} n_{x}\right)=0
$$

The set of three equations consisting of the traction conditions given in Eqs. (3) and the boundary compatibility condition given in Eq. (6) ensures stress functional determinacy on the boundary because three unknown stresses are expressed in terms of three equations.

Equations (2), (3), (5), and (6) represent the CBMF, which ensures the functional determinacy of the stresses both in the field and on the boundary of an elastic continuum. The CBMF can solve a general elastic continuum with stress, displacement, or mixed boundary conditions.

\section{Group III: Continuity Conditions (or Displacement Boundary Conditions)}

The stationary condition of the IFM functional, given by Eq. (48) in the appendix, also yields two displacement boundary conditions, and for the homogeneous case, 


$$
u=\bar{u}=0 \quad(a) \quad v=\bar{v}=0 \quad(b)
$$

where $\bar{u}$ and $\bar{v}$ are prescribed boundary displacements. In the CBMF, the displacement boundary conditions do not appear explicitly in the stress calculations, provided the structure is kinematically stable. The displacements, if required, can be calculated from stresses by integration using the kinematic boundary conditions. $4,6,8,9$

\section{Completed Beltrami-Michell Formulation Solution Strategy for Composite Continuum}

The CBMF solution strategy for a composite elastic continuum with fields of $\Omega_{1}$ and $\Omega_{2}$, and stress, displacement, and boundaries of $\Gamma_{s}, \Gamma_{u}$, and $\Gamma_{t}$, respectively (Fig. 1), are briefly described.

Step 1: Satisfy the field equilibrium and field compatibility conditions given by Eqs. (2) and (4), for both domains $\Omega_{1}$ and $\Omega_{2}$. (In the displacement formulation, the Navier's equations ${ }^{3}$ have to be satisfied.)

Step 2: Satisfy the traction boundary conditions given in Eqs. (3) and boundary compatibility condition given in Eq. (6) on contours $\Gamma_{s}$ and $\Gamma_{u}$, respectively. (In the displacement formulation, equivalent traction conditions written in terms of displacements and displacement boundary conditions on contours $\Gamma_{s}$ and $\Gamma_{u}$, respectively, have to be satisfied.)

Step III: On the interface boundary, $\Gamma_{t}$, three conditions have to be satisfied:

two residual equilibrium equations,

$$
\begin{aligned}
& S_{1}^{I}(\sigma)-S_{2}^{I}(\sigma)=0 \\
& S_{1}^{I I}(\sigma)-S_{2}^{I I}(\sigma)=0
\end{aligned}
$$

and one residual compatibility condition,

$$
\mathbb{R}^{\mathrm{I}}(\sigma)-\mathbb{R}^{\mathrm{II}}(\sigma)=0
$$

The functions $S(\sigma)$ and $\mathscr{R}(\sigma)$ were defined in Eqs. (3) and (6), and the superscripts I and II denote the domains $\Omega_{1}$ and $\Omega_{2}$, respectively. (In the displacement method at the interface boundary, two displacement and two traction continuity conditions have to be satisfied.)

Step 4: Once the solution for stresses has been obtained, displacements, if required, can be calculated by integration. The evaluation of integration constants requires the kinematic boundary conditions. In the Navier's formulation, the displacements must be calculated whether they are required or not. Stresses are then calculated using the differentiation and the stress-strain law. 
The composite structure can be solved by the CBMF or by the Navier's displacement method. The problem, however, cannot be solved by the classical BMF because of the lack of boundary compatibility conditions for the boundary $\Gamma_{u}$ and for the interface contour $\Gamma_{t}$.

\section{Properties of Compatibility Conditions}

Two properties of compatibility conditions for the case of a plane stress problem are given in this section.

(1) The field compatibility condition, written in terms of displacement variables, $u$ and $v$, becomes a trivial constraint, such as an identity $[f(u, v)-f(u, v)]=0$, where $f$ represents the field compatibility condition given by Eq. (4). The boundary compatibility condition given by Eq. (6), however, does not become a trivial equation when written in terms of displacements. In terms of displacements, the boundary compatibility condition given by Eq. (6) becomes

$$
\left[\frac{\partial^{2} v}{\partial x \partial y}-\frac{1}{2}\left(\frac{\partial^{2} u}{\partial x^{2}}+\frac{\partial^{2} v}{\partial x \partial y}\right)\right] n_{x}+\left[\frac{\partial^{2} u}{\partial x \partial y}-\frac{1}{2}\left(\frac{\partial^{2} v}{\partial y^{2}}+\frac{\partial^{2} u}{\partial x \partial y}\right)\right] n_{y}=0
$$

The nontrivial property of the boundary compatibility condition contradicts the popular belief that all compatibility conditions are automatically satisfied in the displacement method.

(2) The field compatibility condition can be derived by eliminating the displacement components from the strain displacement relations. This logic as yet cannot be extended to derive the boundary compatibility condition. At present, the boundary compatibility conditions can be generated only from the IFM variational functional. This is, perhaps, a primary reason why the boundary compatibility conditions could not be formulated earlier.

\section{Applications of the Completed Beltrami-Michell Formulation}

In this section, the CBMF is applied to the stress analysis of circular plates and circular cylindrical shells. Governing equations for both cases are derived from the stationary condition of the IFM functional. Several example problems are presented to demonstrate the CBMF solution procedure.

\section{Completed Beltrami-Michell Formulation for Bending of Circular Plates}

The IFM variational functional for a circular plate subjected to mechanical and thermal loads is given in the appendix. Its stationary condition yields the following equations: 
(a) Field equation of equilibrium:

$$
\frac{d^{2}}{d r^{2}}\left(r M_{r}\right)-\frac{d M_{\varphi}}{d r}+r q=0
$$

(b) Field compatibility condition:

$$
r \frac{d}{d r}\left(M_{\varphi}-v M_{r}\right)+(1+v)\left(M_{\varphi}-M_{r}\right)+K r \frac{\alpha_{t}}{h}\left(\frac{d \Delta t}{d r}\right)=0
$$

In Eqs. (11) and (12), $M_{r}$ and $M_{\varphi}$ are the radial and tangential moment, respectively; $r$ is the radial coordinate; $q$ is the intensity of the distributed load; $h$ is the plate thickness; $K$, a material constant, is defined as $\left(E h^{3} / 12\right)\left(1-v^{2}\right), v$ is the Poisson's ratio; $\alpha_{t}$ is the thermal coefficient of the material; and $\Delta t$ is the temperature difference between the upper and the lower surface of the plate.

(c) Boundary conditions are specialized for various support conditions as follows:

Simply supported contour:

$$
M_{r}=0
$$

Clamped contour:

$$
(1 / K)\left(M_{\varphi}-v M_{r}\right)+\alpha_{t}(\Delta t / h)=0
$$

Note that the condition given in Eq. (13) represents the static boundary condition, whereas Eq. (14) represents the novel boundary compatibility condition.

For the analysis of composite domains, transition (jump) conditions on interfaces between regions made of different materials have to be established. These equilibrium and compatibility conditions at the interface follow:

$$
\begin{gathered}
M_{r}^{\mathrm{I}}=M_{r}^{\mathrm{II}} \\
\frac{d}{d r}\left(r M_{r}^{\mathrm{I}}\right)-M_{\varphi}^{\mathrm{I}}=\frac{d}{d r}\left(r M_{r}^{\mathrm{II}}\right)-M_{\varphi}^{\mathrm{II}} \\
\frac{1}{K^{\mathrm{I}}}\left(M_{\varphi}^{\mathrm{I}}-v^{\mathrm{I}} M_{r}^{\mathrm{I}}\right)+\alpha_{t}^{\mathrm{I}} \frac{\Delta t}{h^{\mathrm{I}}}=\frac{1}{K^{\mathrm{II}}}\left(M_{\varphi}^{\mathrm{II}}-v^{\mathrm{II}} M_{r}^{\mathrm{II}}\right)+\alpha_{t}^{\mathrm{II}} \frac{\Delta t}{h^{\mathrm{II}}}
\end{gathered}
$$

In Eqs. (15), superscripts I and II denote two regions of the composite plate made of different materials. The boundary compatibility condition given by Eq. (14) and the residual boundary compatibility condition at the interface given by Eq. (15c) 
represent new equations for analyzing circular plates. These equations (which were missing from the classical BMF and are unique to the CBMF) make possible the solution of composite plates in terms of stress parameters only.

In the CBMF, the thermal effects are accounted for on the right side of the compatibility conditions given by Eq. (12) and (14), whereas mechanical loads appear on the right side of the equilibrium equation given by Eq. (11). The Navier's displacement method does not include the compatibility conditions in explicit terms, which is the rightful abode for thermal effects. The Navier's formulation, however, accounts for the thermal effects in the equilibrium equations through the concept of work equivalent loads, which may introduce numerical errors when approximate solution techniques are used.

\section{Example: Analysis of a Composite Circular Plate Subjected to Mechanical and Thermal Loads}

The CBMF solution procedure is presented through the analysis of a composite plate (Fig. 2). The plate consists of two segments: an inner plate $\left(\Omega_{i}\right)$ with radius $a$, material properties $E_{i}$ and $v_{i}$, and thickness $h_{i}$; and an outer annular plate $\left(\Omega_{o}\right)$ with inner radius $a$, outer radius $b$, material properties $E_{o}$ and $v_{o}$, and thickness $h_{o}$. The inner plate $\left(\Omega_{i}\right)$ is subjected to a uniformly distributed mechanical load of intensity $q$, and the outer plate $\left(\Omega_{o}\right)$ is exposed to uneven heating with the temperature difference $\Delta t$. The plate is clamped at the outer contour, given by $r=b$. This example illustrates the CBMF solution process for (a) using the boundary compatibility condition at a clamped contour, (b) analyzing composite domains by means of transition conditions, and (c) analyzing thermomechanical loads.

Equations (11) and (12) are solved to obtain general expressions for the moments $M_{r}$ and $M_{\varphi}$ for the regions $\Omega_{i}$ and $\Omega_{o}$, respectively:

$$
\begin{gathered}
M_{r}^{i}(r)=-\frac{B_{1}}{r^{2}}+\frac{1}{2} C_{1}\left(1+v_{i}\right) \log r+\frac{1}{4} C_{1}\left(1-v_{1}\right)+\frac{1}{2} D_{1}-\frac{1}{16}\left(3+v_{i}\right) q r^{2} \\
M_{\varphi}^{i}(r)=\frac{B_{1}}{r^{2}}+\frac{1}{2} C_{1}\left(1+v_{i}\right) \log r-\frac{1}{4} C_{1}\left(1-v_{1}\right)+\frac{1}{2} D_{1}-\frac{1}{16}\left(1+3 v_{i}\right) q r^{2} \\
M_{r}^{o}(r)=-\frac{B_{2}}{r^{2}}+\frac{1}{2} C_{2}\left(1+v_{o}\right) \log r+\frac{1}{4} C_{2}\left(1-v_{o}\right)+\frac{1}{2} D_{2} \\
M_{\varphi}^{o}(r)=\frac{B_{2}}{r^{2}}+\frac{1}{2} C_{2}\left(1+v_{o}\right) \log r-\frac{1}{4} C_{2}\left(1-v_{o}\right)+\frac{1}{2} D_{2}
\end{gathered}
$$


where $B_{1}, C_{1}, D_{1}, B_{2}, C_{2}$, and $D_{2}$ are integration constants.

The thermal load does not appear in the homogeneous solution given by Eqs. (17a) and (17b) because $\frac{d}{d r}(\alpha \Delta t)=0$. These six constants are calculated from the following six conditions: one boundary compatibility condition given in Eq. (14) at the outer contour $(r=b)$, three transition conditions given in Eqs. (15) at the interface $(r=a)$, and (3) two implicit conditions at the origin ( $r=0$ ). The implicit conditions require that the moments $M_{r}$ and $M_{\varphi}$ have the finite values at the origin. The solution is obtained for a specific composite plate with the inner plate $\left(\Omega_{i}\right)$ made of aluminum and the outer plate $\left(\Omega_{o}\right)$ made of steel. Numerical values for the material parameters in the domains $\Omega_{i}$ and $\Omega_{o}$, respectively, are taken as $E_{i}=10.6 \times 10^{6} \mathrm{psi}, v_{i}=0.33$, $a_{t}^{(i)}=12.6 \times 10^{6} /{ }^{\circ} \mathrm{F}, E_{o}=30.0 \times 10^{6} \mathrm{psi}, v_{o}=0.30$, and $a_{t}^{(o)}=6.3 \times 10^{-6} /{ }^{\circ} \mathrm{F}$. The radii are $a=6$ in. and $b=12$ in., and the thicknesses are $h_{i}=0.2$ in. and $h_{o}=0.15$ in.; the magnitude of the distributed load is $q=100 \mathrm{lb} / \mathrm{in}^{2}{ }^{2}$; and the temperature difference is $\Delta t=50^{\circ} \mathrm{F}$. After the integration constants are determined, the final solution for $M_{r}$ and $M_{\varphi}$ is for the domain $\Omega_{i}$ $(0 \leq r \leq a)$

$$
\begin{aligned}
& M_{r}^{i}(r)=844.05-20.81 r^{2} \\
& M_{\varphi}^{i}(r)=844.05-12.44 r^{2}
\end{aligned}
$$

and for the domain $\Omega_{o}(a \leq r \leq b)$

$$
\begin{aligned}
& M_{r}^{o}(r)=2046.63-\left(5203.06 / r^{2}\right)-1170 \log r \\
& M_{\varphi}^{o}(r)=2676.63+\left(5203.06 / r^{2}\right)-1170 \log r
\end{aligned}
$$

The displacements, if required, can be obtained by integrating the moment-curvative relations and by using displacement continuity conditions to evaluate constants of integration. Displacements for the domains $\Omega_{q}$ and $\Omega_{0}$, respectively, are given as

$$
\begin{gathered}
w^{i}(r)=3.1209-0.0356 r^{2}+0.1757 \times 10^{-3} r^{4} \\
w^{o}(r)=5.3614-0.1344 r^{2}-0.7296 \log r+0.04417 r^{2} \log r
\end{gathered}
$$

The solution for $M_{r}, M_{\varphi}$, and $w$ (Eqs. (18) to (20)), respectively, has been verified from the corresponding solution with the Navier's displacement method of analysis.

\section{Integrated Force Method Variational Formulation for Cylindrical Shells}

In this section, through an example of a circular cylindrical shell subjected to thermomechanical loads, the CBMF is extended to analyzing shell structures wherein membrane and bending responses are coupled. In CBMF, $M_{x}$ and force $N_{\varphi}$ are 
the force unknowns for this problem. The variational functional for the problem is defined, and all equations of the CBMF, including the novel boundary compatibility conditions, for the cylindrical shell are obtained from its stationary condition. The IFM functional for the problem has the following form:

$$
\Pi_{s}^{c}=A+B-W
$$

where the strain energy $A$, the complementary energy $B$, and the work of external load $W$ are given as

$$
\begin{gathered}
A=\int_{\Omega}\left[M_{x}\left(-\frac{d^{2} w}{d x^{2}}+(1+v) \alpha_{\mathrm{t}} \frac{\Delta t}{h}\right)+N_{\varphi}\left(-\frac{w}{a}+\alpha_{t} t_{o}\right)\right] d \Omega \\
B=\int_{\Omega}\left[\Psi\left(\frac{M_{x}}{K}+(1+v) \alpha_{t} \frac{\Delta t}{h}\right)-a \frac{d^{2} \Psi}{d x^{2}}\left(\frac{N_{\varphi}}{E h}+\alpha_{t} t_{o}\right)\right] d \Omega \\
W=\int_{\Omega} q w d \Omega
\end{gathered}
$$

where $w$ is the radial displacement; $K=\left(E h^{3} / 12\right)\left(1-v^{2}\right)$ is the rigidity; $t_{o}$ is the temperature at the midsurface of the shell; $\Delta t$ is the temperature difference between the inner and outer surfaces; and $\Psi$ is the stress function. The stress function is defined through a procedure similar to that given by Washizu, ${ }^{10}$ as

$$
\begin{gathered}
M_{x}=\Psi \\
N_{\varphi}=-a \frac{d^{2} \Psi}{d x^{2}}+q
\end{gathered}
$$

The variation $\Pi_{s}^{c}$ has the following form:

$$
\begin{aligned}
\delta \Pi_{s}^{c}= & -\int_{\Omega}\left[\frac{d^{2} M_{x}}{d x^{2}}+\frac{1}{a} N_{\varphi}+q\right] d \Omega \delta w \\
& -\int_{\Omega}\left[\frac{M_{x}}{K}-\frac{a}{E h} \frac{d^{2} N_{\varphi}}{d x^{2}}+(1+v) \alpha_{t} \frac{\Delta t}{h}-a \alpha_{t} \frac{d^{2} t_{o}}{d x^{2}}\right] d \Omega \delta \Psi \\
& +2 a \pi\left[M_{x} \delta\left(-\frac{d w}{d x}\right)+\frac{d M_{x}}{d x} \delta w\right]_{x_{a}}^{x_{b}}+2 a \pi\left[a\left(\frac{N_{\varphi}}{E h}+\alpha_{t^{t}}\right) \delta \frac{d \Psi}{d x}-a\left(\frac{1}{E h} \frac{d N_{\varphi}}{d x}+\alpha_{t} \frac{d t_{o}}{d x}\right) \delta \Psi\right]_{x_{a}}^{x_{b}}
\end{aligned}
$$


The stationary condition of the variational functional with respect to the displacement $w$ and the stress function $\Psi$ yields all the equations of the CBMF as follows:

(a) Field equation of equilibrium:

$$
\frac{1}{a} N_{\varphi}+\frac{d^{2} M_{x}}{d x^{2}}+q=0
$$

(b) Field compatibility condition:

$$
\frac{M_{x}}{K}-\frac{a}{E h} \frac{d^{2} N_{\varphi}}{d x^{2}}+(1+v) \alpha_{t} \frac{\Delta t}{h}-a \alpha_{t} \frac{d^{2} t_{o}}{d x^{2}}=0
$$

Contour terms in Eq. (24) yield boundary conditions. These are specialized for various support conditions as follows.

\section{Free Contour}

On a free contour, $w \neq 0$ and $\frac{d}{d x} w \neq 0$, hence both the moment and its derivative must vanish:

$$
\begin{gathered}
M_{x}=0 \\
\frac{d}{d x}\left(M_{x}\right)=0
\end{gathered}
$$

\section{Simply Supported Contour}

The rotation of the cross section is not prevented on a simply supported boundary. The condition $\delta(d w / d r) \neq 0$ results in

$$
M_{x}=0
$$

Because $w \neq 0$, the derivative $\frac{d}{d x}\left(M_{x}\right)=d \Psi / d x$ is not zero on a simply supported boundary, resulting in

$$
\left(N_{\varphi} / E h\right)+\alpha_{t} t_{o}=0
$$

Equation (28b) represents a boundary compatibility condition for a simply supported boundary for the cylindrical shell.

\section{Clamped Contour}

For the clamped contour, both displacements and rotations are equal to zero, and on such a boundary two compatibility conditions must be satisfied. The boundary compatibility conditions, which are the coefficient of $\delta d \Psi d x$ and $d \Psi$ in the contour forms in Eq. (24), follow:

$$
\begin{aligned}
& \left(N_{\varphi} / E h\right)+\alpha_{t} t_{o}=0 \\
& \frac{1}{E h} \frac{d N_{\varphi}}{d x}+\alpha_{t} \frac{d t_{o}}{d x}=0
\end{aligned}
$$


Transition conditions, used to analyze composite shells, are derived similarly as those for circular plates. They consist of two residual equilibrium conditions

$$
\begin{gathered}
M_{x}^{(\mathrm{I})}-M_{x}^{(\mathrm{II})}=0 \\
\frac{d}{d x} M_{x}^{(\mathrm{I})}+\frac{d}{d x} M_{x}^{(\mathrm{II})}=0
\end{gathered}
$$

and two residual boundary compatibility conditions given as

$$
\begin{gathered}
\left(\frac{N_{\varphi}^{(\mathrm{I})}}{E_{1} h_{1}}+\alpha_{t}^{\mathrm{I}} t_{o}\right)-\left(\frac{N_{\varphi}^{(\mathrm{II})}}{E_{2} h_{2}}+\alpha_{t}^{\text {II } t_{o}}\right)=0 \\
\left(\frac{1}{E_{1} h_{1}} \frac{d N_{\varphi}^{(\mathrm{I})}}{d x}+\alpha_{t}^{\mathrm{I}} \frac{d t_{o}}{d x}\right)+\left(\frac{1}{E_{2} h_{2}} \frac{d N_{\varphi}^{(\mathrm{II})}}{d x}+\alpha_{t}^{\mathrm{II}} \frac{d t_{o}}{d x}\right)=0
\end{gathered}
$$

The field equations given in Eqs. (25) and (26), together with appropriate boundary conditions represent the number of equations sufficient to solve the shell bending problem for stresses $M_{x}$ and $N_{\varphi}$. The boundary compatibility conditions given in Eqs. (29) are derived for the first time. Two boundary compatibility conditions given in Eqs. (29) have to be imposed on a clamped boundary, and one compatibility condition given in Eq. (28b) must be satisfied on a simply supported boundary. Note that without the boundary compatibility conditions the solution $\left(M_{x}\right.$ and $\left.N_{\varphi}\right)$ for the shell bending problems cannot be obtained for problems with either the displacement boundary conditions given in Eqs. (28) or the mixed boundary conditions given in Eqs. (29). The transition conditions given in Eqs. (30c) and (30d) enable the solution of composite shells by the CBMF.

The field equations given by Eqs. (25) and (26) may be uncoupled to obtain the following alternative systems:

$$
\begin{gathered}
\frac{d^{4} M_{x}}{d x^{4}}+4 \beta^{4} M_{x}=-\frac{d^{2} q}{d x^{2}}-\frac{E h}{a^{2}} \alpha_{t}\left[(1+v) \frac{\Delta t}{h}+a \frac{d^{2} t_{o}}{d x^{2}}\right] \\
N_{\varphi}=-a\left(\frac{d^{2} M_{x}}{d x^{2}}+q\right)
\end{gathered}
$$

or

$$
\frac{d^{4} N_{\varphi}}{d x^{4}}+4 \beta^{4} N_{\varphi}=-\frac{E h}{K a} q+\frac{E h}{a} \alpha_{t}\left[(1+v) \frac{1}{h} \frac{d^{2} \Delta t}{d x^{2}}-a \frac{d^{4} t_{o}}{d x^{4}}\right]
$$




$$
M_{x}=\frac{K a}{E h} \frac{d^{2} N_{\varphi}}{d x^{2}}+K \alpha_{t}\left[(1+v) \frac{\Delta t}{h}-a \frac{d^{2} t_{o}}{d x^{2}}\right]
$$

where $\beta^{4}=3\left(1-v^{2}\right) / a^{2} h^{2}$. Note that both Eqs. (31a) and (32a) are fourth-order equations, and either one can be selected for solution. Here, the moment equation (31a) is selected, and its general solution has the following form:

$$
M_{x}=C_{1} \cosh \beta x+C_{2} \sinh \beta x+C_{3} \cos \beta x+C_{4} \sin \beta x+\Psi_{p}^{(q)}+\Psi_{p}^{(\Delta t)}
$$

where $C_{1}, C_{2}, C_{3}$, and $C_{4}$ are the constants of integration, and $\Psi_{p}^{(q)}$ and $\Psi_{p}{ }^{(\Delta t)}$ are particular integrals for distributed loads and temperature, respectively. The constants of integration are obtained by imposing appropriate boundary conditions. Once $M_{x}$ is known, $N_{\varphi}$ can be calculated by back substitution from Eq. (31b). The solutions for two examples are provided to illustrate the CBMF solution process. The first example is a short cylindrical shell subjected to thermomechanical loads. The second example is a composite shell with clamped and simply supported boundary conditions.

\section{Example 1: Analysis of a Short Cylindrical Shell}

A simply supported cylindrical shell made of isotropic material with length $L$ and radius $a$ is shown in Fig. 3. The origin of the coordinate system is located at the centroid of the shell. The analysis is performed for two cases: (1) a uniformly distributed load and (2) uneven heating with $\Delta t$ such that $t_{o}=0$. The material and the geometric parameters of the shell are such that the product $\beta L<5$; hence, it must be analyzed as a short shell. The general solution for each case is obtained by substituting the particular integrals into the general solution given by Eq. (33) and then by imposing the boundary conditions for simply supported contours at $x= \pm L / 2$ to evaluate the constants.

\section{Solution for the Mechanical Load}

For this case, $\Psi_{p}^{(q)}=0$ and $\Psi_{p}^{(\Delta t)}=0$. The solutions obtained for $M_{x}$ and $N_{\varphi}$ after solving for the integration constants in Eq. (33) using simply supported boundary conditions at $x= \pm L / 2$ have the following form:

$$
\begin{gathered}
M_{x}(x)=\left(q / 2 \beta^{2} D\right)\left(g_{2} \cosh \beta x \cos \beta x-g_{1} \sinh \beta x \sin \beta x\right) \\
N_{\varphi}(x)=(q a / D)\left[g_{2}\left(g_{2}-\sinh \beta x \sin \beta x\right)+g_{1}\left(g_{1}-\cosh \beta x \cos \beta x\right)\right]
\end{gathered}
$$

where $D=g_{1}^{2}+g_{2}^{2}, g_{1}=\cosh \lambda \cos \lambda, g_{2}=\sinh \lambda \sin \lambda ;$ and $\lambda=\beta L / 2$. If required, the displacement $w$ may be calculated using the stress-strain relations as 


$$
w(x)=\left(-q a^{2} / D E h\right)\left[g_{2}\left(g_{2}-\sinh \beta x \sin \beta x\right)+g_{1}\left(g_{1}-\cosh \beta x \cos \beta x\right)\right]
$$

\section{Solution for the Thermal Load}

For this case, particular integrals $\Psi_{p}^{(q)}=0$ and $\Psi_{p}^{(\Delta t)}=-K(1+v) a_{t} \Delta t / h$, and the final expressions for the internal forces take the following form:

$$
\begin{gathered}
M_{x}(x)=\left(-\Psi_{p}^{(\Delta t)} / D\right)\left[g_{1}\left(g_{1}-\cosh \beta x \cos \beta x\right)+g_{2}\left(g_{2}-\sinh \beta x \cos \beta x\right)\right] \\
N_{\varphi}(x)=2 \beta^{2}\left(\Psi_{p}^{(\Delta t)} / D\right)\left(g_{2} \cosh \beta x \cos \beta x-g_{1} \sinh \beta x \sin \beta x\right)
\end{gathered}
$$

If required, $w$ can be calculated as

$$
w(x)=-2\left(a \beta^{2} \Psi_{p}^{(\Delta t)} / E D h\right)\left(g_{2} \cosh \beta x \cos \beta x-g_{1} \sinh \beta x \sin \beta x\right)
$$

The solution for this simple problem required a boundary compatibility condition (Eq. (28b)), even for a simply supported boundary, and hence the example could not have been solved by classical BMF.

\section{Example 2: Analysis of a Long Composite Shell}

A composite cylindrical shell of radius $a$ and length $2 L$ is shown in Fig. 4. This shell is composed of two regions with different material and geometrical properties. Region I, bounded by contours 1-1 and 2-2, has material parameters $E_{1}$ and $v_{1}$, and thickness $h_{1}$; and region II, bounded by contours 2-2 and 3-3, has material and geometric properties $E_{2}, v_{2}$, and $h_{2}$. The shell is clamped along contour 1-1, and simply supported along the contour 3-3. Both regions are subjected to a uniformly distributed load of intensity $q$. Region I is also subjected to a temperature change of $\Delta t$.

Total solution for the composite shell is obtained by superposing the two component solutions. Each component solution involves four integration constants ( $C_{1}$ to $C_{4}$ in Eq. (33)); hence, there are a total of eight unknowns for the composite shell. The eight constants of integration are evaluated from the following eight conditions: two boundary compatibility conditions for the clamped boundary 1-1 (see Fig. 4) given in Eqs. (29), two boundary conditions for the simple supported boundary 3-3 given in Eqs. (28), and four transition conditions at the interface 2-2 given in Eqs. (30).

For simplicity, the long shell condition (the products of $\beta_{1} L$ and $\beta_{2} L$ ) is assumed for both components. Consequently, the response for the composite shell can be obtained by superposing effects from the three boundaries: that is, from the simply supported boundary, fixed boundary, and interface boundary as shown in Fig. 4. 


\section{Response From the Fixed Boundary (Contour 1-1)}

The local coordinate system is defined such that the axis $x_{1}$ is placed along the axis of the shell, with the origin in the plane defined by contour 1-1 (see Fig. 4). The solution process is similar to that presented for the short shell, given as

$$
\begin{gathered}
M_{x}\left(x_{1}\right)=\left(q / 2 \beta_{1}^{2}\right) e^{-\beta_{1} x_{1}}\left(\sin \beta_{1} x_{1}-\cos \beta_{1} x_{1}\right)+\Psi_{p}^{(\Delta t)} \\
N_{\varphi}\left(x_{1}\right)=a q\left[e^{-\beta_{1} x_{1}}\left(\cos \beta_{1} x_{1}+\sin \beta_{1} x_{1}\right)-I\right]
\end{gathered}
$$

Using Eq. (38b), the expression for the displacement is obtained as

$$
w\left(x_{1}\right)=\left(-q a^{2} / E h_{1}\right)\left[\left(e^{-\beta_{1} x_{1}}\left(\cos \beta_{1} x_{1}+\sin \beta_{1} x_{1}\right)-1\right]\right.
$$

\section{Response From the Interface (Contour 2-2)}

The expressions for $M_{x}, N_{\varphi}$, and $w$, defined for regions I and II, respectively, are obtained as

$$
\begin{gathered}
M_{x}^{(\mathrm{I})}\left(x_{2}\right)=e^{-\beta_{1} x_{2}}\left(A_{1} \cos \beta_{1} x_{2}+B_{1} \sin \beta_{1} x_{2}\right)+\Psi_{p}^{(\Delta t)} \\
N_{\varphi}^{(\mathrm{I})}\left(x_{2}\right)=2 \beta_{1}^{2} e^{-\beta_{1} x_{2}}\left(-B_{1} \cos \beta_{1} x_{2}+A_{1} \sin \beta_{1} x_{2}\right) \\
w^{(\mathrm{I})}=(-1 / a E h) 2 \beta_{1}^{2} e^{-\beta_{1} x_{2}}\left(-B_{1} \cos \beta_{1} x_{2}+A_{1} \sin \beta_{1} x_{2}\right) \\
M_{x}^{(\mathrm{II})}\left(x_{3}\right)=e^{-\beta_{2} x_{3}}\left(A_{2} \cos \beta_{2} x_{3}+B_{2} \sin \beta_{2} x_{3}\right) \\
N_{\varphi}^{(\mathrm{II})}\left(x_{3}\right)=2 \beta_{2}^{2} e^{-\beta_{2} x_{3}}\left(-B_{2} \cos \beta_{2} x_{3}+A_{2} \sin \beta_{2} x_{3}\right) \\
w^{(\mathrm{II})}=(-1 / a E h) 2 \beta_{2}^{2} e^{-\beta_{2} x_{3}}\left(-B_{2} \cos \beta_{2} x_{3}+A_{2} \sin \beta_{2} x_{3}\right)
\end{gathered}
$$

where $A_{1}, B_{1}, A_{2}$, and $B_{2}$ are the constants of integration, and $x_{2}$ and $x_{3}$ are defined separately for each region (Fig. 4). Four constants of integration are calculated by imposing four transition conditions given in Eqs. (30) along the interface contour 2-2. The four transition conditions yield the following four equations to compute four constants of integration:

$$
\begin{gathered}
A_{1}-A_{2}=-\Psi_{p}^{(\Delta t)} \\
\beta_{1}\left(B_{1}-A_{1}\right)+\beta_{2}\left(B_{2}-A_{2}\right)=0 \\
-2 \frac{\beta_{1}^{2}}{E_{1} h_{1}} B_{1}+2 \frac{\beta_{2}^{2}}{E_{2} h_{2}} B_{2}=\frac{q}{E_{2} h_{2}}-\frac{q}{E_{1} h_{1}} \\
\frac{\beta_{1}^{3}}{E_{1} h_{1}}\left(A_{1}+B_{1}\right)+\frac{\beta_{2}^{3}}{E_{2} h_{2}}\left(A_{2}+B_{2}\right)=0
\end{gathered}
$$

The solution of Eqs. (42) yields the four integration constants: 


$$
\begin{aligned}
& A_{1}=(1 / \bar{D})\left[\Psi_{p}^{(\Delta t)} \beta_{2}^{2}\left(-2 k \beta_{1} \beta_{2}-k \beta_{1}^{2}-k^{2} \beta_{2}^{2}\right)+(q / 2)(1-k)\left(k \beta_{2}^{2}-\beta_{1}^{2}\right)\right] \\
& A_{2}=(1 / \bar{D})\left[\Psi_{p}^{(\Delta t)} \beta_{1}^{2}\left(\beta_{1}^{2}+k \beta_{2}^{2}+2 k \beta_{1} \beta_{2}\right)+(q / 2)(1-k)\left(k \beta_{2}^{2}-\beta_{1}^{2}\right)\right] \\
& B_{1}=(1 / \bar{D})\left[\Psi_{p}^{(\Delta t)} k \beta_{2}^{2}\left(\beta_{1}^{2}-k \beta_{2}^{2}\right)+\left(q / 2 \beta_{1}\right)(1-k)\left(2 k \beta_{2}^{3}+k \beta_{1} \beta_{2}^{2}+\beta_{1}^{3}\right)\right] \\
& B_{2}=(1 / \bar{D})\left[\Psi_{p}^{(\Delta t)} \beta_{1}^{2}\left(\beta_{1}^{2}-k \beta_{2}^{2}\right)-\left(q / 2 \beta_{2}\right)(1-k)\left(2 \beta_{1}^{3}+\beta_{1}^{2} \beta_{2}+k \beta_{2}^{3}\right)\right]
\end{aligned}
$$

where $\bar{D}=2 k \beta_{1} \beta_{2}\left(\beta_{1}^{2}+\beta_{2}^{2}\right)+\left(\beta_{1}^{2}+k \beta_{2}^{2}\right)^{2}$ and $K=\left(E_{1} h_{1}\right) /\left(E_{2} h_{2}\right)$. The integration constants given in Eqs. (43) are introduced into Eqs. (40) and (41) to obtain $M_{x}$ and $N_{\varphi}$ for both regions I and II. Then, displacement $w$, which can be calculated following the procedure given earlier, has the following form:

\section{Response From Simply Supported Conditions (Contour 3-3)}

For this case, a procedure similar to that presented for the clamped edge effects along contour 1-1 is followed. The coordinate axis $x_{4}$ is defined as shown in Fig. 4. Contour 3-3 is simply supported, and the conditions given in Eqs. (28) are applied to obtain the expressions for the internal forces:

$$
\begin{aligned}
& M_{x}\left(x_{4}\right)=\left(q / 2 \beta_{2}^{2}\right) e^{-\beta_{2} x_{4}} \sin \beta_{2} x_{4} \\
& N_{\varphi}\left(x_{4}\right)=q a\left(e^{-\beta_{2} x_{4}} \cos \beta_{2} x_{4}-1\right)
\end{aligned}
$$

and $w$ is calculated as

$$
w\left(x_{4}\right)=\left(q a^{2} / E h_{2}\right)\left(1-e^{-\beta_{2} x_{4}} \cos \beta_{2} x_{4}\right)
$$

As mentioned earlier, solution for any point is obtained by superposition of the expansions given by Eqs. (38) to (45). Analysis of the composite shell with simply supported, clamped, and interface boundaries can be obtained using CBMF. The problem, however, cannot be solved using classical BMF because of the following missing boundary compatibility conditions: Eqs. (29a) and (29b) for the clamped edge, Eq. (28b) for the simply supported boundary, and Eqs. (30c) and (30d) for the interface boundary.

\section{Conclusions}

The completed Beltrami-Michell Formulation (CBMF), wherein stresses are considered as the primary variables, is obtained by augmenting the classical Beltrami-Michell Formulation (BMF) with novel boundary compatibility conditions. The 
CBMF, which can analyze stress, displacement, and mixed boundary value problems in elasticity, alleviates the limitations of the classical formulation, which could analyze only stress boundary value problems. All equations of the CBMF for analyzing circular plates and cylindrical shells subjected to both mechanical and thermal loads have been derived from the stationary condition of the IFM variational functional. Transition conditions required for analyzing composite plates and shells made of different materials have been established. The CBMF has been used to solve several stress, displacement, and mixed boundary value problems in elasticity. In CBMF, displacements, if required, can be calculated from stresses by back substitutions. The CBMF is a true alternative to the Navier's displacement formulation. 


\section{Appendix - Variational Formulation for CBMF}

This appendix briefly describes the derivations of the CBMF governing equations for problems of two-dimensional elasticity and bending of circular plates.

\section{Governing Equations for Two-dimensional Elasticity}

The variational functional of the IFM for a two-dimensional domain $\Omega$ bounded by the contour $\Gamma$ has the following form

where

$$
\Pi_{p}^{p s}=A+B-W
$$

$$
\begin{gathered}
A=h \int_{\Omega}\left[\sigma_{x} \frac{\partial u}{\partial x}+\sigma_{y} \frac{\partial u}{\partial y}+\tau_{x y}\left(\frac{\partial u}{\partial y}+\frac{\partial v}{\partial x}\right)\right] d \Omega \\
B=h \int_{\Omega}\left[\frac{\partial^{2} \Phi}{\partial y^{2}} \frac{\sigma_{x}-v \sigma_{y}}{E}+\frac{\partial^{2} \Phi}{\partial x^{2}} \frac{\sigma_{y}-v \sigma_{x}}{E} \frac{\partial^{2} \Phi}{\partial x \partial y} \frac{2(1+v)}{E} \tau_{x y}\right] d \Omega \\
W=h \int_{\Omega}\left(B_{x} u+B_{y} v\right) d \Omega-\int_{\Gamma_{1}}\left(\bar{P}_{x} u+\bar{P}_{y} v\right) d \Gamma_{1}-\int_{\Gamma_{2}}\left(P_{x} \bar{u}+P_{y} \bar{v}\right) d \Gamma_{2}
\end{gathered}
$$

The variation of the functional with respect to $u, v$, and $\Phi$ is given as

$$
\begin{aligned}
\delta \Pi_{p}^{p s}= & h \int_{\Omega}\left[\left(\frac{\partial \sigma_{x}}{\partial_{x}}+\frac{\partial \tau_{x y}}{\partial_{y}}+B_{x}\right) \delta u+\left(\frac{\partial \tau_{x y}}{\partial x}+\frac{\partial \sigma_{y}}{\partial y}+B_{y}\right) d v\right] d \Omega \\
& +\frac{h}{E} \int_{\Omega}\left[\frac{\partial^{2}}{\partial x^{2}}\left(\sigma_{y}-v \sigma_{x}\right)+\frac{\partial^{2}}{\partial y^{2}}\left(\sigma_{x}-v \sigma_{y}\right)-2(1+v) \frac{\partial^{2} \tau_{x y}}{\partial x \partial y}\right] d \Omega \delta \Phi \\
& -h \int_{\Gamma_{1}}\left[\left(\sigma_{x} n_{x}+\tau_{x y} n_{y}-\bar{P}_{x}\right) \partial u+\left(\tau_{x y} n_{x}+\sigma_{y} n_{y}-\bar{P}_{y}\right) \delta v\right] d \Gamma_{1} \\
& +h \int_{\Gamma_{2}}\left[(u+\bar{u}) \delta\left(\sigma_{x} n_{x}+\tau_{x y} n_{y}\right)+(v+\bar{v})\left(\tau_{x y} n_{x}+\sigma_{y} n_{y}\right)\right] d \Gamma_{2} \\
& +\frac{h}{E} \int_{\Gamma_{2}}\left[\frac{\partial}{\partial x}\left(\sigma_{x}-v \sigma_{y}\right) n_{x} \frac{\partial}{\partial y}\left(\sigma_{y}-v \sigma_{x}\right) n_{y}-2(1+v)\left(\frac{\partial \tau_{x y}}{\partial x} n_{y}+\frac{\partial \tau_{x y}}{\partial y} n_{x}\right)\right] d \Gamma_{2} \delta \Phi
\end{aligned}
$$


In Eqs. (47) and (48), $\Gamma_{1}$ and $\Gamma_{2}$ are portions of the boundary, where traction and kinematic boundary conditions, respectively, are imposed; $B_{x}$ and $B_{y}$ are components of body forces; an overbar denotes prescribed quantities; and $\Phi$ is the Airy's stress function. The stationary condition of the variational functional yields the governing equations of the CBMF given in Eqs. (2), (3), (5), and (6).

\section{Governing Equations for Bending of Circular Plates}

For a circular plate subjected to distributed loads $q$ and the temperature change $\Delta t$,

$$
\begin{gathered}
A=2 \pi \int_{r_{a}}^{r_{b}}\left[M_{r}\left(-\frac{d^{2} w}{d r^{2}}\right)+M_{\varphi}\left(-\frac{1}{r} \frac{d w}{d r}\right)\right] r d r \\
B=2 \pi \int_{r_{a}}^{r_{b}}\left[\Psi\left(\frac{M_{r}-v M_{\varphi}}{K}+\alpha_{t} \frac{\Delta t}{h}\right)+\frac{d}{d r}(r \Psi)\left(\frac{M_{\varphi}-v M_{r}}{K}+\alpha_{\mathrm{t}} \frac{\Delta t}{h}\right)\right] r d r \\
W=2 \pi \int_{r_{a}}^{r_{b}} q w r d r
\end{gathered}
$$

where $r_{a}$ and $r_{b}$ are the radial coordinates of the plate.

The variation of the IFM functional with respect to variables $w$ and $\Psi$ can be written as

$$
\begin{aligned}
\delta \Pi_{p}^{c}= & 2 \pi\left\{-\int_{r_{a}}^{r_{b}}\left[\frac{d^{2}}{d r^{2}}\left(r M_{r}\right)-\frac{d M_{\varphi}}{d r}+r q\right] d r \delta w\right. \\
& +\int_{r_{a}}^{r_{b}}\left[\frac{M_{r}-v M_{\varphi}}{K}-\frac{d^{2}}{d r^{2}}\left(r \frac{M_{\varphi}-v M_{r}}{K}+\frac{\alpha_{t}}{h} \frac{d \Delta t}{d r}\right)\right] r d r \delta \Psi \\
& \left.+\left[r M_{r}\left(-\delta \frac{d w}{d r}\right)\right]_{r_{a}}^{r_{b}}+\left[\left(\frac{d}{d r}\left(r M_{r}\right)-M_{\varphi}\right) \delta w\right]_{r_{a}}^{r_{b}}+\left[r^{2}\left(\frac{M_{\varphi}-v M_{r}}{K}+\alpha_{t} \frac{\Delta t}{h}\right) \delta \Psi\right]_{r_{a}}^{r_{b}}\right\}
\end{aligned}
$$

The stationary condition of the functional yields the CBMF equations for a circular plate given in Eqs. (11) to (14). 


\section{References}

${ }^{1}$ Love, A. E. H., A Treatise on the Mathematical Theory of Elasticity, Dover, New York, 1944.

${ }^{2}$ Timoshenko, S., and Goodier, J. N., Theory of Elasticity, McGraw Hill, New York, 1951.

${ }^{3}$ Sokolnikoff, I. S., Mathematical Theory of Elasticity, 2nd ed., McGraw Hill, New York, 1956.

${ }^{4}$ Patnaik, S. N., and Satish, H., "Analysis of Continuum Using the Boundary Compatibility Conditions of Integrated Force Method," Computers \& Structures, Vol. 34, No. 2, 1990, pp. 287-295.

5Patnaik, S. N., "The Variational Energy Formulation for the Integrated Force Method," AIAA Journal, Vol. 24, No. 1, 1986, pp. 129-136.

6Patnaik, S.N., and Nagaraj, M. S., “Analysis of Continuum by the Integrated Force Method, " Computers \& Structures, Vol. 25, No. 6, 1987, pp. 899-905.

${ }^{7}$ Patnaik, S.N., Guptill, J., and Berke,L., "Singularity in Structural Optimization," International Journal for Numerical Methods in Engineering, Vol. 36, No. 67, 1993, pp. 931-944.

${ }^{8}$ Vijayakumar, K., Murty, A. V. K., and Patnaik, S. N., "Basis for the Analysis of Solid Continuum by the Integrated Force Method," AIAA Journal, Vol. 26, No. 5, 1988, pp. 626-629.

9Murty, A. V. K., Vijayakumar, K., and Patnaik, S. N., "Studies on Integrated Force Method," Indian Space Research Organization Report, ISRO-IISc (STC) 15a-1988/1, ISc., Bangalore, India, 1988.

${ }^{10}$ Washizu, K., Variational Methods in Elasticity and Plasticity, Pergamon Press, Oxford, England, 1968. 


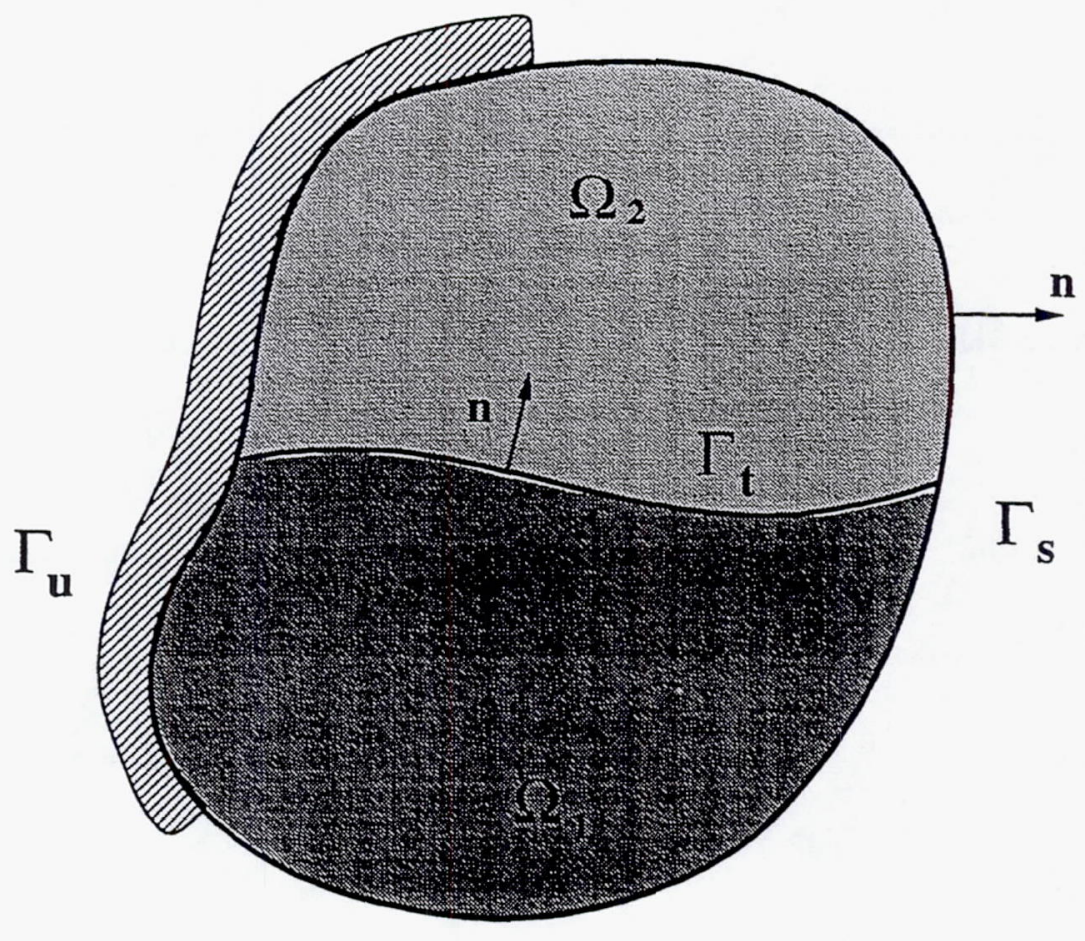

Fig. 1 Composite elastic continuum 


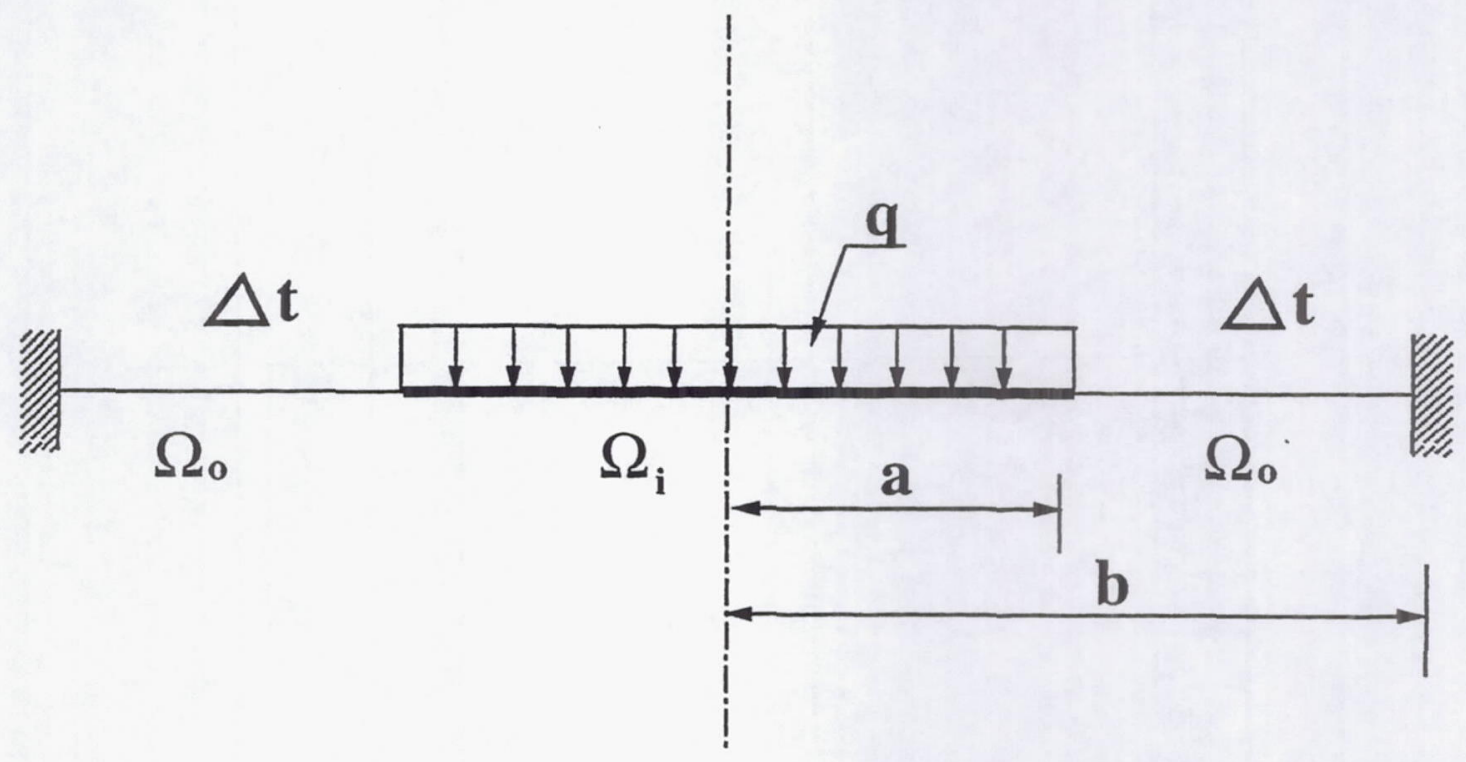

Fig. 2 Composite circular plate subjected to a uniform load, $q$, and temperature, $\Delta t$. 


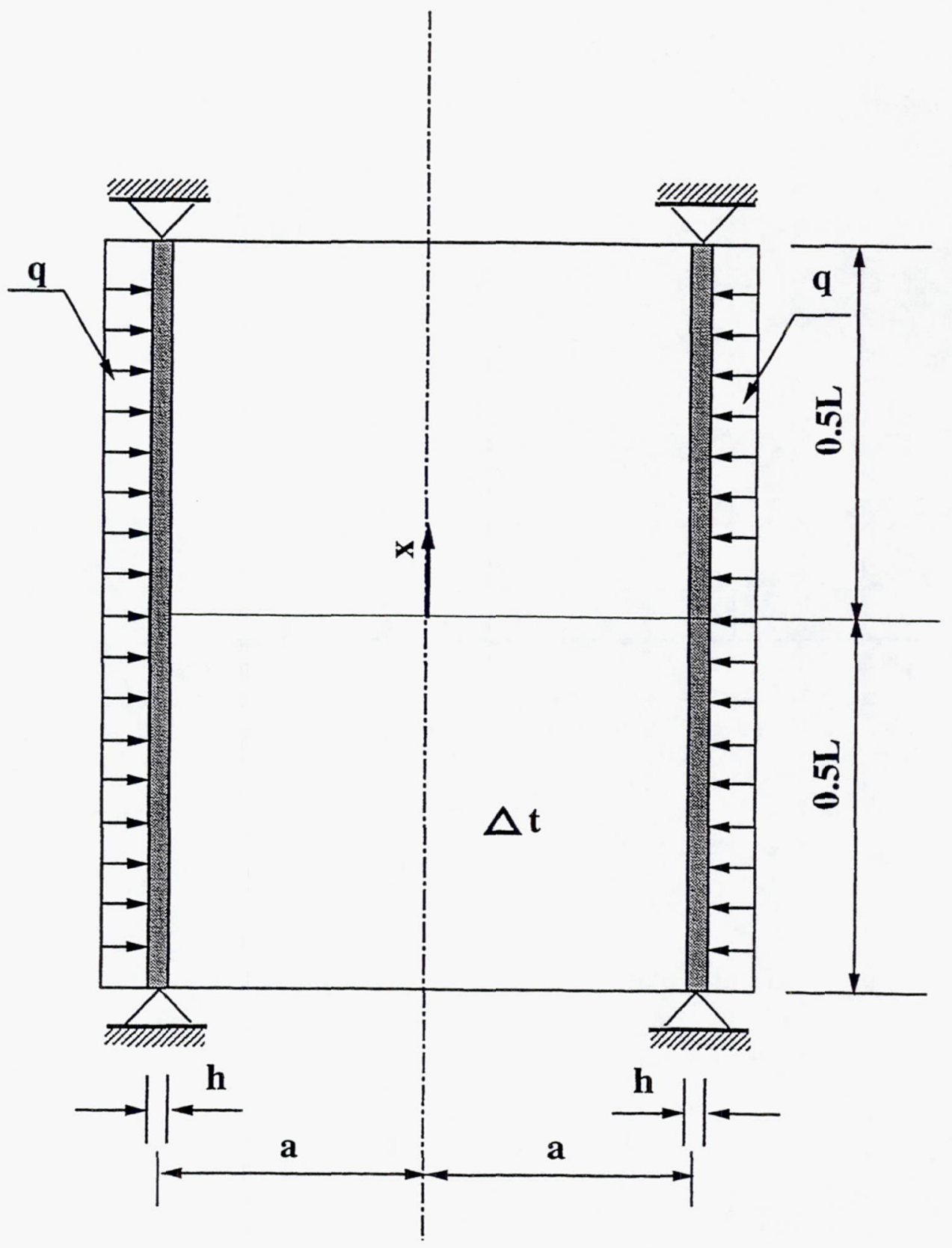

Fig. 3 Short, circular cylindrical shell. 


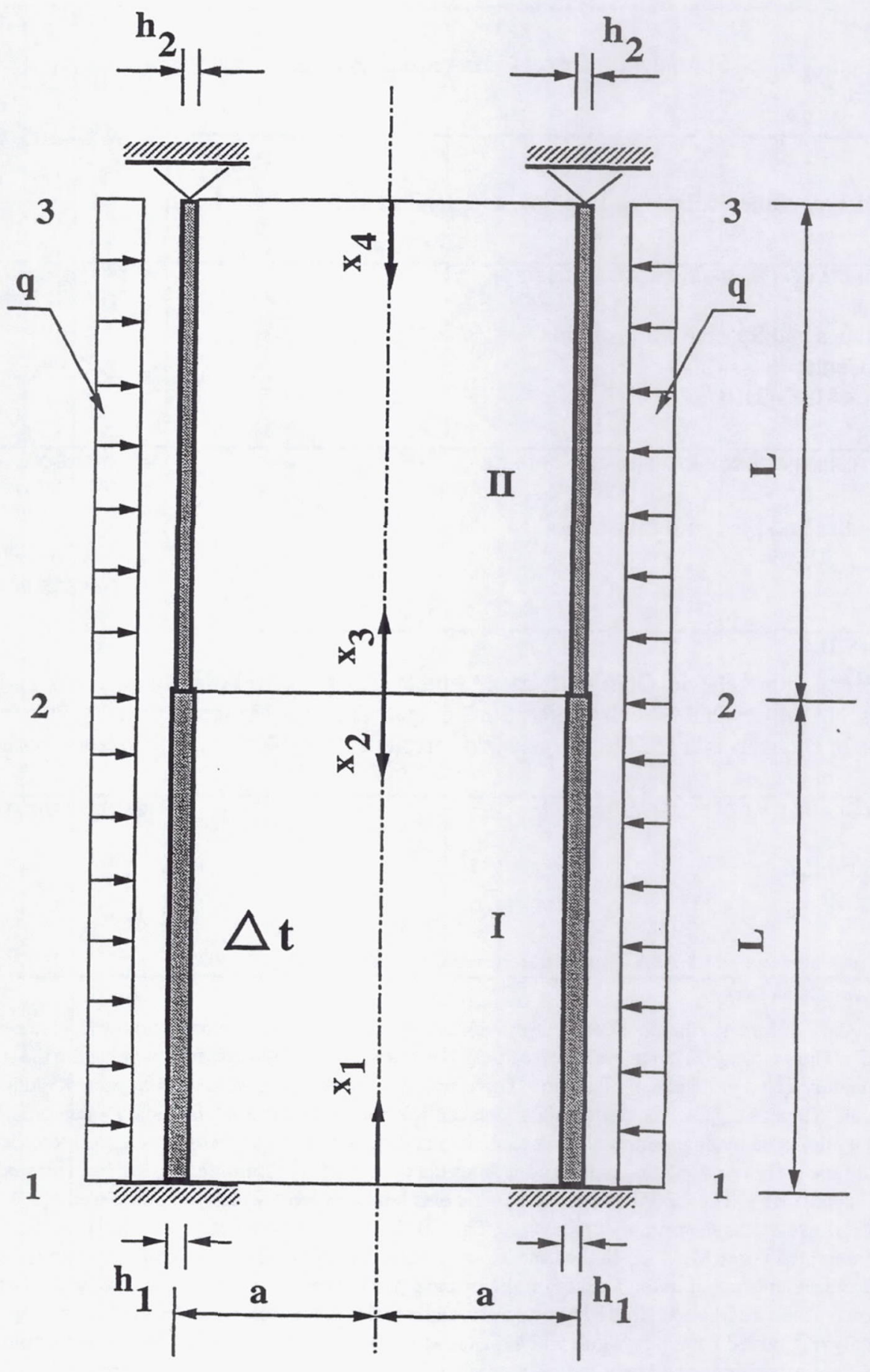

Fig. 4 Long, composite, circular cylindrical shell. 
Public reporting burden for this collection of information is estimated to average 1 hour per response, including the time for reviewing instructions, searching existing data sources, gathering and maintaining the data needed, and completing and reviewing the collection of information. Send comments regarding this burden estimate or any other aspect of this collection of information, including suggestions for reducing this burden, to Washington Headquarters Services, Directorate tor In Davis Highway, Suite 1204, Arlington, VA 22202-4302, and to the Office of Management and Budget, Paperwork Reduction Project (0704-0188), Washington, DC 20503.

\begin{tabular}{|l|l|l|}
\hline 1. AGENCY USE ONLY (Leave blank) & $\begin{array}{c}\text { 2. REPORT DATE } \\
\text { September 1995 }\end{array}$ & $\begin{array}{r}\text { 3. REPORT TYPE AND DATES COVERED } \\
\text { Technical Memorandum }\end{array}$
\end{tabular}

\section{TITLE AND SUBTITLE}

Completed Beltrami-Michell Formulation for Analyzing Mixed Boundary Value Problems in Elasticity

6. AUTHOR(S)

WU-505-63-53

Surya N. Patnaik, Igor Kaljevic, Dale A. Hopkins, and Sunil Saigal

7. PERFORMING ORGANIZATION NAME(S) AND ADDRESS(ES)

8. PERFORMING ORGANIZATION REPORT NUMBER

National Aeronautics and Space Administration

Lewis Research Center

Cleveland, Ohio 44135-3191

E-9296

9. SPONSORING/MONITORING AGENCY NAME(S) AND ADDRESS(ES)

10. SPONSORING/MONITORING AGENCY REPORT NUMBER

National Aeronautics and Space Administration

Washington, D.C. 20546-0001

NASA TM-106809

Corrected Copy

\section{SUPPLEMENTARY NOTES}

Surya N. Patnaik and Igor Kaljevic, Ohio Aerospace Institute, 22800 Cedar Point Road, Brook Park, Ohio 44142;

Dale A. Hopkins, NASA Lewis Research Center; Sunil Saigal, Carnegie Mellon University, Department of Civil Engineering, Pittsburgh, Pennsylvania 15213. Responsible person, Dale A. Hopkins, organization code 5210, (216) 433-3260.

12a. DISTRIBUTIONAVAILABILITY STATEMENT

12b. DISTRIBUTION CODE

Unclassified -Unlimited

Subject Category 39

This publication is available from the NASA Center for Aerospace Information, (301) 621-0390.

13. ABSTRACT (Maximum 200 words)

In elasticity, the method of forces, wherein stress parameters are considered as the primary unknowns, is known as the Beltrami-Michell Formulation (BMF). The existing BMF can only solve stress boundary value problems; it cannot handle the more prevalent displacement of mixed boundary value problems of elasticity. Therefore, this formulation, which has restricted application, could not become a true altemative to the Navier's displacement method, which can solve all three types of boundary value problems. The restrictions in the BMF have been alleviated by augmenting the classical formulation with a novel set of conditions identified as the boundary compatibility conditions. This new method, which completes the classical force formulation, has been termed the Completed BeltramiMichell Formulation (CBMF). The CBMF can solve general elasticity problems with stress, displacement, and mixed boundary conditions in terms of stresses as the primary unknowns. The CBMF is derived from the stationary condition of the variational functional of the Integrated Force Method. In the CBMF, stresses for kinematically stable structures can be obtained without any reference to the displacements either in the field or on the boundary. This paper presents the CBMF and its derivation from the variational functional of the Integrated Force Method. Several examples are presented to demonstrate the applicability of the completed formulation for analyzing mixed boundary value problems under thermomechanical loads. Selected example problems include a cylindrical shell, wherein membrane and bending responses are coupled, and a composite circular plate.

14. SUBJECT TERMS

Force method; Completed Beltrami-Michell formulation; Boundary compatibility conditions; Elastic continua, Thermomechanical stress analysis

15. NUMBER OF PAGES 29

16. PRICE CODE

$\mathrm{A} 03$

\begin{tabular}{|c|c|}
\hline $\begin{array}{l}\text { 17. SECURTY CLASSIFICATION } \\
\text { OF REPORT }\end{array}$ & $\begin{array}{c}\text { 18. SECURTY CLASSIFICATION } \\
\text { OF THIS PAGE } \\
\text { Unclassified }\end{array}$ \\
\begin{tabular}{l} 
Unclassified \\
\hline
\end{tabular}
\end{tabular}

19. SECURITY CLASSIFICATION

20. LIMITATION OF ABSTRACT

Unclassified

Unclassified

OF ABSTRACT Unclassified 